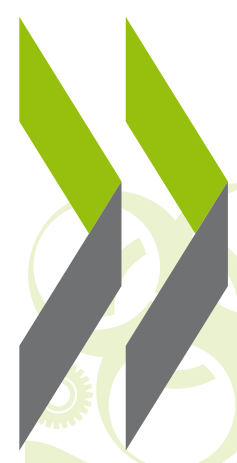

\title{
Investissements en faveur des compétences de la population adulte immigrée
}

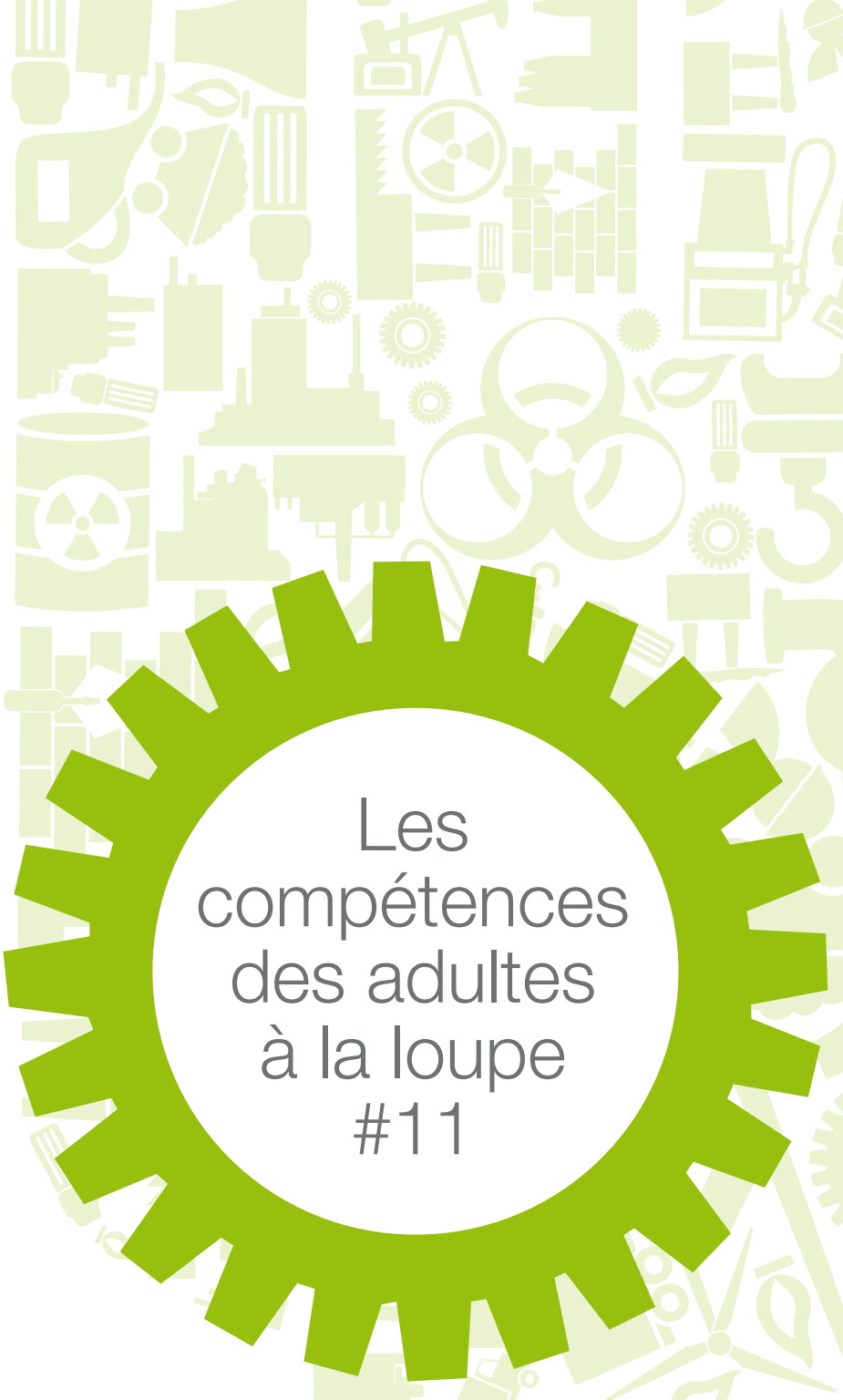




\section{LES COMPÉTENCES DES ADULTES}

\section{ÁLA LOUPE}

- Les adultes nés à l'étranger participent moins aux activités d'apprentissage tout au long de la vie que les adultes autochtones. Les différences sont faibles, mais s'expliquent en grande partie par des facteurs sociodémographiques, tels que l'âge, le niveau d'éducation et les compétences en littératie.

- En règle générale, les immigrés souhaiteraient bénéficier de davantage de formation pour adultes. Pour se former, ils se heurtent à des obstacles plus importants, mais ils y consacrent davantage de temps lorsqu'ils parviennent à accéder à une formation.

- Les immigrés ont moins la possibilité de pratiquer leurs compétences en littératie dans leur cadre professionnel, même après contrôle des différences relatives aux caractéristiques individuelles et aux professions.

L’apprentissage tout au long de la vie est un élément crucial des politiques liées aux compétences et au marché du travail. En raison des progrès technologiques rapides, les demandes en matière de compétences évoluent, ce qui impose une actualisation de plus en plus fréquente des compétences des travailleurs. En raison du prolongement de leur carrière professionnelle, les travailleurs risquent davantage de voir leurs compétences devenir obsolètes, car ils sont plus susceptibles de voir évoluer les demandes en matière de compétences ou font davantage face à un déclin de leurs capacités cognitives en raison de leur âge.

Il est d'autant plus nécessaire pour les immigrés de participer aux formations pour adultes. En moyenne, les adultes nés à l'étranger ont un niveau de compétences en littératie et en numératie qui est inférieur à celui de leurs pairs autochtones (voir « Pourquoi les adultes immigrés sont-ils moins compétents en littératie que leurs pairs autochtones? ", Les compétences des adultes à la loupe, $n^{\circ}$ 6) et la réussite de leur intégration dans leur pays d'accueil dépend fortement de leur acquisition de la langue de ce pays. De plus, les compétences acquises par les adultes nés à l'étranger dans leur pays d'origine ne concordent que rarement avec celles qui sont requises sur le marché du travail du pays d'accueil.

\section{L'écart de participation aux activités d'apprentissage tout au long de la vie est faible entre les adultes nés à l'étranger et les adultes autochtones}

Nonobstant ces considérations, les données de l'Évaluation des compétences des adultes (PIAAC) montrent que les adultes nés à l'étranger étaient moins susceptibles, en comparaison avec leurs pairs autochtones, de participer à des activités d'éducation et de formation formelles et non formelles au cours des douze mois précédant l'enquête. L'écart de niveau de participation s'élève à 4 points de pourcentage en moyenne et est plus marqué en Estonie (16 points de pourcentage), en Allemagne (14 points de pourcentage) et en Slovénie (12 points de pourcentage) (Graphique 1). Cependant, dans près de la moitié des pays et des économies qui ont participé à l'enquête PIAAC, l'écart n'est pas important sur le plan statistique. L'écart est principalement lié aux différences de caractéristiques socio-démographiques (telles que l'âge, le sexe, la situation au regard de l'emploi et les compétences en littératie) qui sont elles-mêmes des variables prédictives probantes de la participation aux formations pour adultes. De plus, les différences de niveau de participation entre les adultes nés à l'étranger et leurs pairs autochtones au sein des pays sont extrêmement faibles par rapport aux différences entre pays : le niveau général de participation atteignait pour sa valeur la plus faible $20 \%$ en Grèce et pour sa valeur la plus élevée 67 \% en Nouvelle-Zélande. La corrélation entre les pays des taux de participation des adultes nés à l'étranger et des adultes autochtones s'élève à $91 \%$, ce qui semble indiquer que le pays de résidence est un facteur qui détermine beaucoup plus le niveau de participation que le statut au regard de l'immigration. 


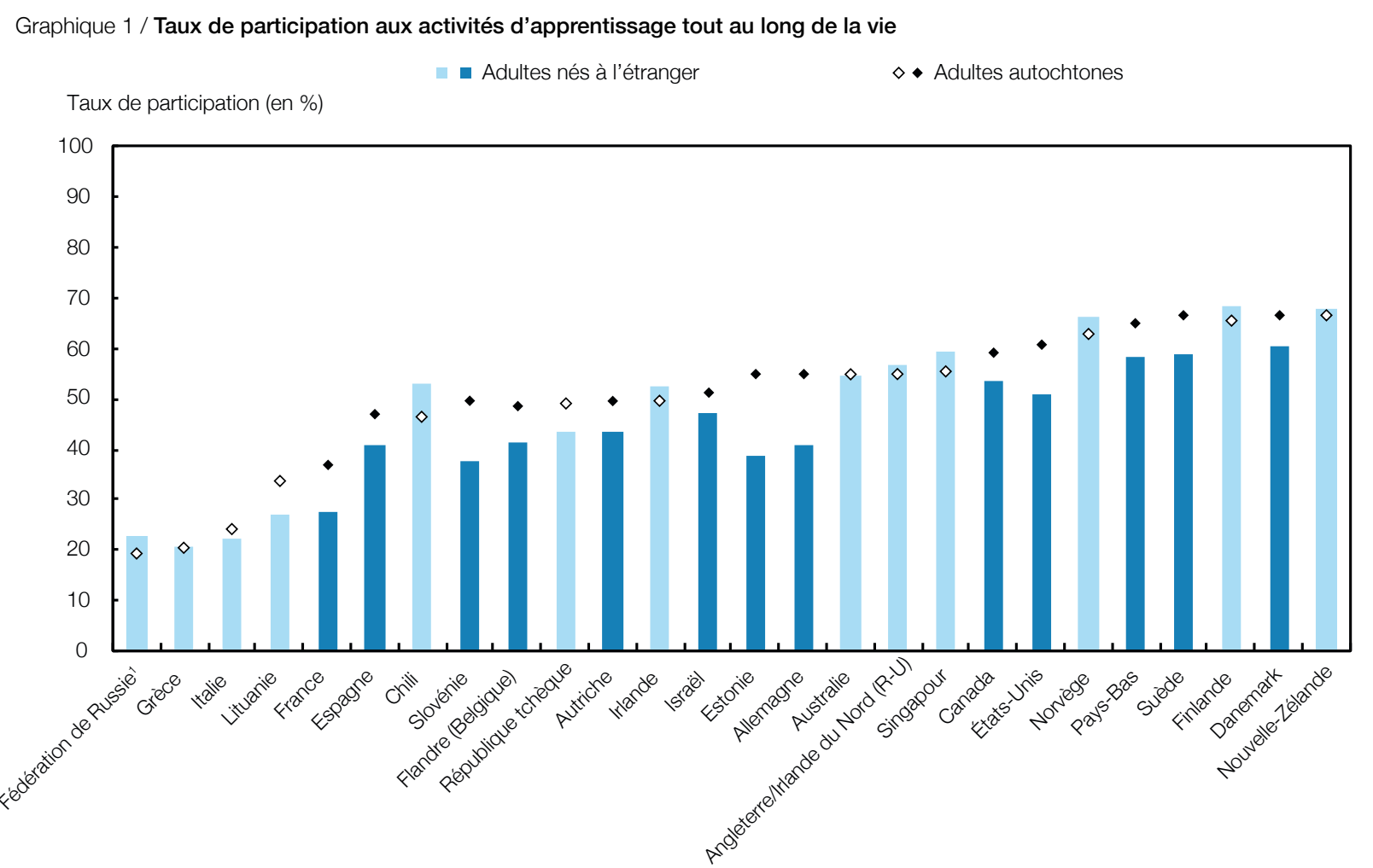

Remarque : Les pays dans lesquels les différences de taux de participation entre les adultes nés à l'étranger et les adultes autochtones sont statistiquement proches de zéro sont indiqués par une couleur plus claire.

1 L'échantillon pour la Fédération de Russie ne couvre pas la population de la municipalité de Moscou.

Les pays et économies sont classés par ordre croissant du taux de participation global.

Source : Évaluation des compétences des adultes (PIAAC) (2012, 2015), www.oecd.org/skills/piaac/data/

\section{Les immigrés ne sont pas en mesure de participer autant qu'ils le voudraient aux activités de formation}

Bien que les différences de taux de participation aux formations pour adultes ne soient pas très importantes entre les adultes nés à l'étranger et les adultes autochtones, cela ne signifie pas que tous les travailleurs bénéficient de suffisamment d'activités de formation. Dans le cadre de l'évaluation PIAAC, il a été demandé aux participants s'ils n'avaient pas pu prendre part à des formations qui les intéressaient et quels avaient été les obstacles à cette participation. Dans la vaste majorité des pays et des économies, les adultes nés à l'étranger ont été plus susceptibles, en comparaison avec les adultes autochtones, d'indiquer que leurs demandes en matière de formation n'avaient pas été satisfaites (graphique 2). 


\section{LES COMPÉTENCES DES ADULTES}

\section{ÀLA LOUPE}

Graphique 2 / Obstacles à la participation à des activités de formation

- Adultes autochtones

Pourcentage

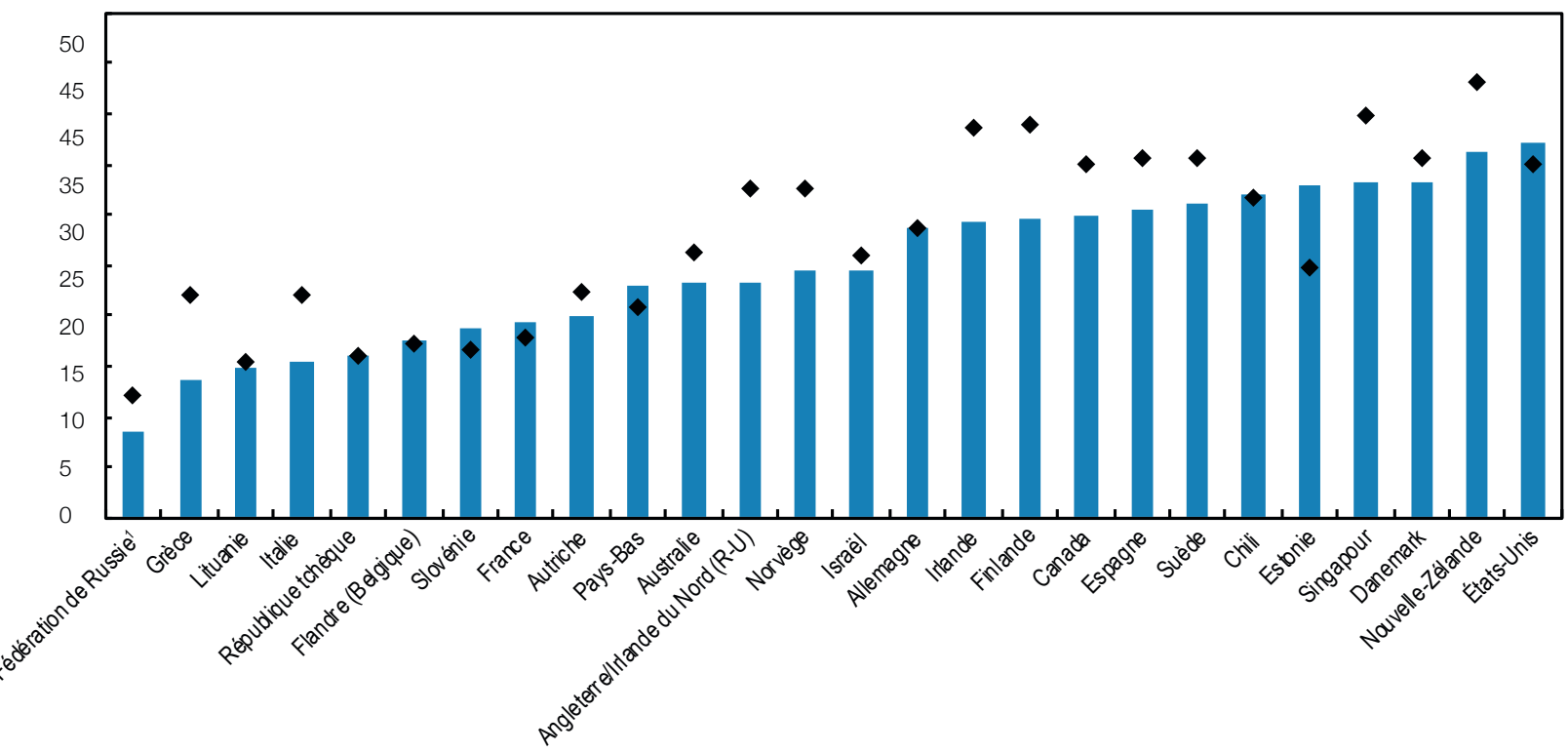

Remarque : Le graphique représente le pourcentage d'adultes ayant indiqué qu'ils voulaient participer à une activité d'apprentissage au cours des douze mois précédant l'entretien, mais qui, en fin de compte, n'ont pas commencé cette activité (adultes qui ont rencontré un obstacle). ${ }^{1}$ L'échantillon pour la Fédération de Russie ne couvre pas la population de la municipalité de Moscou.

Les pays et économies sont classés par ordre croissant du pourcentage d'adultes autochtones qui se sont heurtés à un obstacle. Source : Évaluation des compétences des adultes (PIAAC) (2012, 2015), www.oecd.org/skills/piaac/data/

Même après contrôle de diverses caractéristiques observables, les immigrés sont davantage susceptibles de faire part de l'existence d'obstacles à leur participation dans près de la moitié des pays et économies participants. L'écart ajusté atteint jusqu'à 12 points de pourcentage en Finlande et entre 7 et 8 points de pourcentage en Angleterre/Irlande du Nord (Royaume-Uni), en Grèce, en Italie et en Norvège.

Lorsqu'il leur a été demandé d'indiquer les obstacles à leur participation aux activités de formation, les adultes nés à l'étranger ont été plus susceptibles, en comparaison avec les adultes autochtones, d'indiquer qu'il s'agissait essentiellement d'obstacles liés à leurs responsabilités familiales et à leurs ressources financières.

Les données montrent par ailleurs que les adultes nés à l'étranger qui peuvent accéder à une formation consacrent davantage d'heures en moyenne à ces activités que les adultes autochtones. Dans les pays tels que le Danemark, la Finlande, la Flandre (Belgique) et les Pays-Bas, ces différences sont importantes (valeurs comprises entre 40 et $60 \%$ ), ce qui fait apparaître que les adultes nés à l'étranger ont une demande (latente) plus forte en matière de formation.

\section{Les immigrés ont moins de possibilité de pratiquer leurs compétences en littératie}

Pour perfectionner leurs compétences ou éviter leur déclin, les adultes n'ont pas seulement besoin de suivre des formations formelles ou informelles, mais doivent aussi pouvoir mettre leurs compétences en pratique. L'enquête PIAAC recueille des informations sur la fréquence à laquelle les adultes réalisent certaines tâches dans le cadre professionnel ou prennent part à certaines activités dans leur vie quotidienne, qui nécessitent le recours à des compétences en lecture, en écriture, en numératie ou en technologies de l'information et de la communication (TIC). Les données montrent que les adultes nés à l'étranger ont généralement moins l'occasion de pratiquer leurs compétences en lecture dans le cadre professionnel (Graphique 3). Dans une grande mesure, cela s'explique par des différences liées à des caractéristiques observables (telles que leur niveau d'éducation ou leurs compétences en littératie), qui incitent les adultes nés à l'étranger et les adultes autochtones à s'orienter vers des professions différentes nécessitant des compétences distinctes. Cependant, même après contrôle de ces facteurs (y compris la profession), l'écart reste assez important, notamment dans des pays tels que l'Allemagne, l'Autriche, l'Italie et la Slovénie. Cela peut s'expliquer par l'existence de pratiques discriminatoires (qui font, par exemple, que les travailleurs immigrés se voient confier systématiquement 
des tâches plus simples, alors qu'ils pourraient assumer des tâches plus complexes) ou d'aspects liés aux compétences des travailleurs nés à l'étranger qui ne transparaissent pas dans les données, mais que les employeurs peuvent observer.

Graphique 3 / Différences relatives à l'utilisation des compétences en lecture dans le cadre professionnel entre les adultes nés à l'étranger et les adultes autochtones

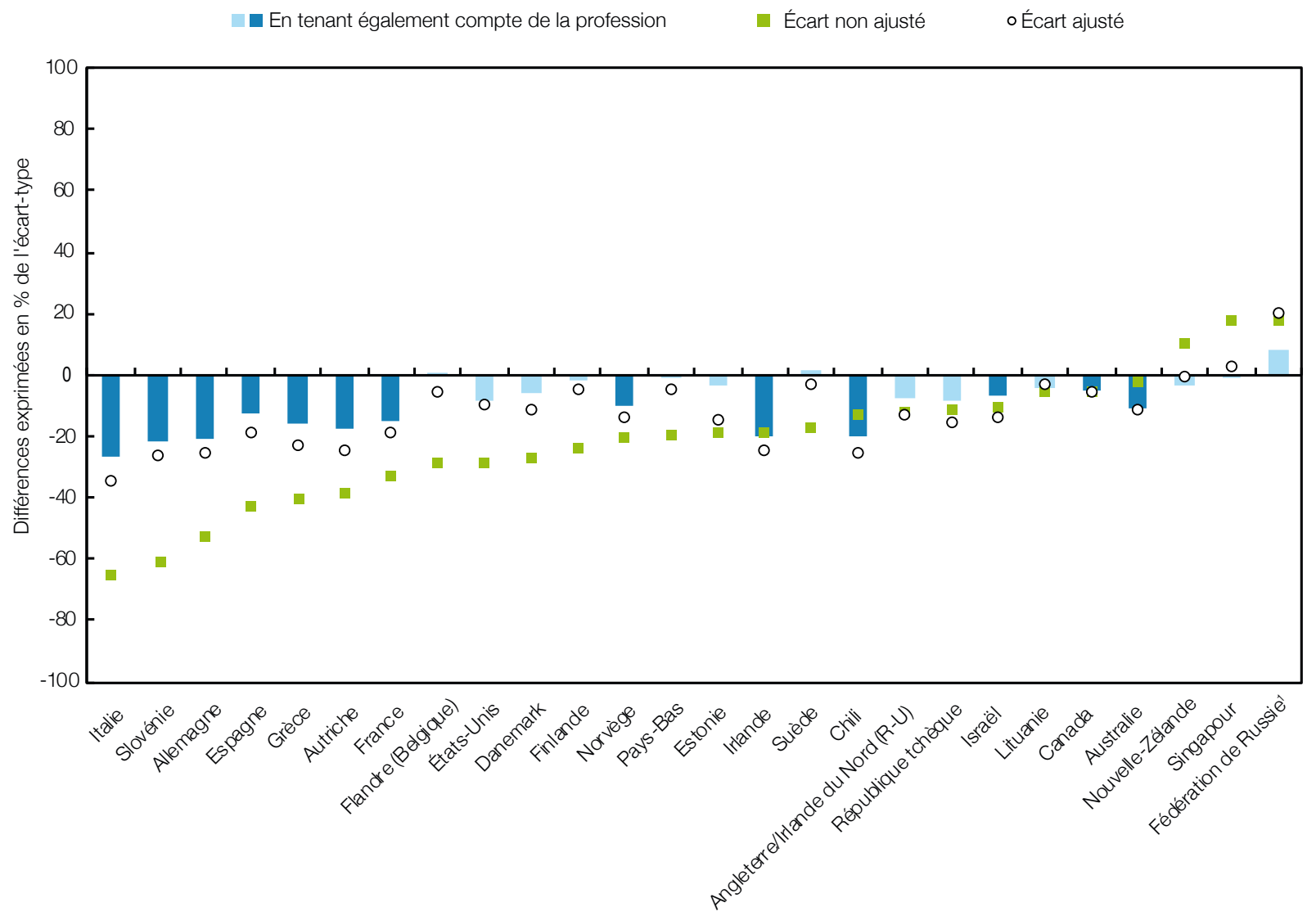

Remarques : Le graphique montre les différences estimées entre les adultes nés à l'étranger et les adultes autochtones concernant l'indice d'utilisation des compétences en lecture dans le cadre professionnel. Les écarts ajustés tiennent compte des différences liées à l'âge, à l'âge au carré, au sexe, au niveau de formation, aux compétences en littératie et au statut au regard de l'emploi. Les barres tiennent également compte de variables fictives pour les professions avec une classification à 1 chiffre (CITP 2008). Les différences statistiquement significatives sont indiquées par une couleur plus claire.

${ }^{1}$ L'échantillon pour la Fédération de Russie ne couvre pas la population de la municipalité de Moscou.

Les pays et les économies sont classés en fonction d'un écart non ajusté.

Source : Évaluation des compétences des adultes (PIAAC) (2012, 2015), www.oecd.org/skills/piaac/datal. 


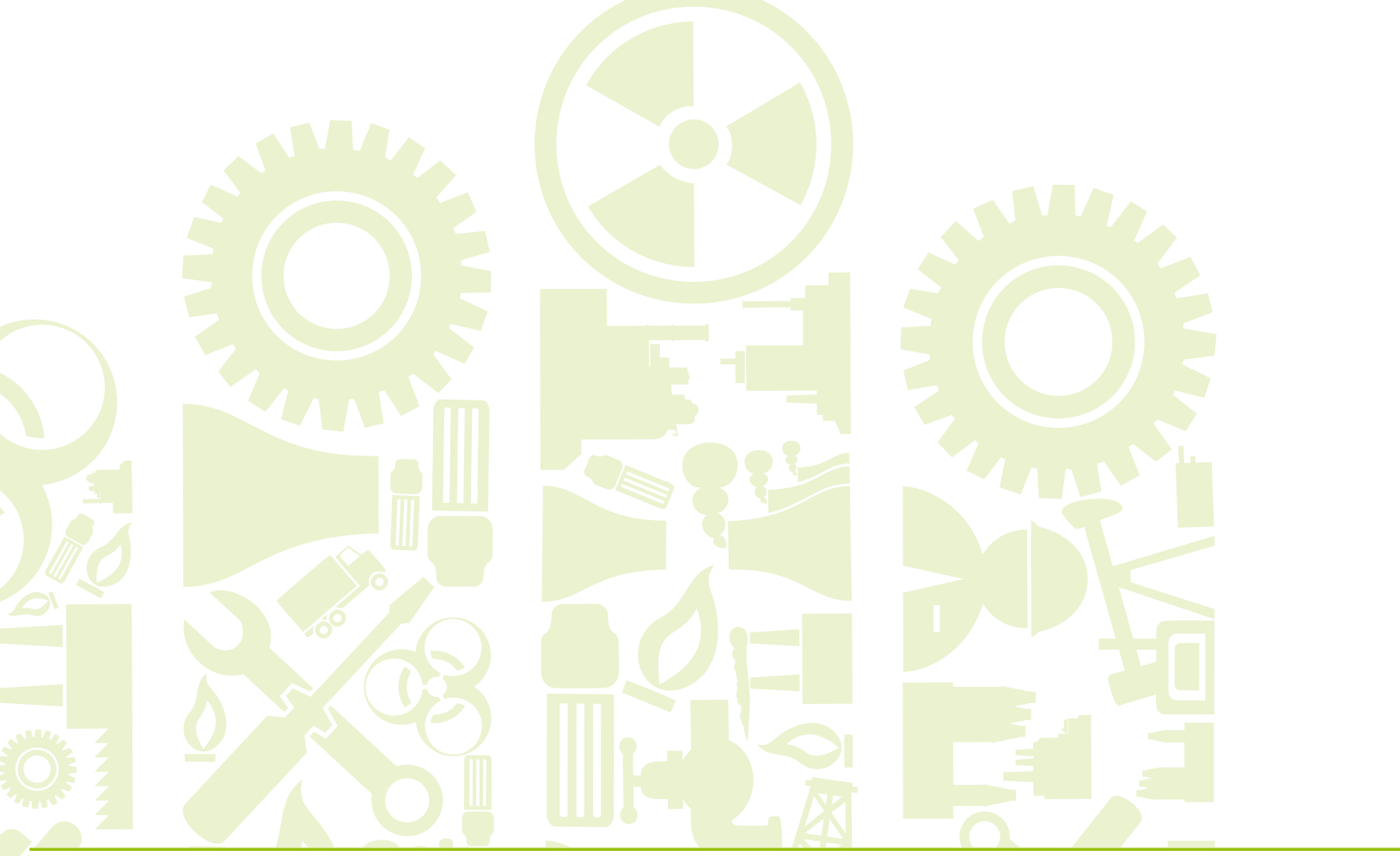

\section{Pour

L'apprentissage tout au long de la vie est particulièrement important pour les immigrés, qui sont souvent défavorisés sur le plan de la langue et des compétences valorisées sur le marché du travail de leur pays d'accueil. Cependant, les adultes nés à l'étranger sont moins susceptibles, en comparaison avec leurs pairs autochtones, de participer à des formations et se heurtent à des obstacles financiers et non financiers plus importants pour prendre part à ces formations. II convient de déployer des efforts politiques visant non seulement à multiplier les possibilités de formation mais aussi à éliminer les obstacles empêchant la participation à ces activités.

\section{> CONTACT : $\quad$ Marco Paccagnella (Marco.Paccagnella@oecd.org); (edu.piaac@oecd.org) \\ > POUR EN OECD (2018), Skills on the Move: Migrants in the Survey of Adult Skills, \\ SAVOIR PLUS : $\quad$ Études de l'OCDE sur les compétences, OECD Publishing, Paris, https://doi.org/10.1787/9789264307353-en}

OECD (2017), «Pourquoi les adultes immigrés sont-ils moins compétents en littératie que leurs pairs autochtones ?», Les compétences des adultes à la loupe, No. 6, OECD Publishing, Paris, https://doi.org/10.1787/bc5c81e1-fr.

$>$ VISITER :

\section{www.oecd.org/skills/piaac}

Indicateurs de l'éducation à la loupe - PISA à la loupe - L'enseignement à la loupe

\footnotetext{
L'Évaluation des compétences des adultes est un produit du Programme de l'OCDE pour l'évaluation internationale des compétences des adultes (PIAAC).
}

Ce document est publié sous la responsabilité du Secrétaire général de l'OCDE. Les opinions qui y sont exprimées et les arguments qui y sont employés ne reflètent pas nécessairement les vues officielles des pays membres de l'OCDE.

Ce document et toute carte qu'il peut comprendre sont sans préjudice du statut de tout territoire, de la souveraineté s'exerçant sur ce dernier, du tracé des frontières et limites internationales, et du nom de tout territoire, ville ou région.

Vous êtes autorisés à copier, télécharger ou imprimer du contenu OCDE pour votre utilisation personnelle. Vous pouvez inclure des extraits des publications, des bases de données et produits multimédia de l'OCDE dans vos documents, présentations, blogs, sites Internet et matériel d'enseignement, sous réserve de faire mention de la source OCDE et du copyright. Les demandes pour usage public ou commercial ou de traduction devront être adressées à pubrights@oecd.org.

Les données statistiques concernant Israël sont fournies par et sous la responsabilité des autorités israéliennes compétentes. L'utilisation de ces données par l'OCDE est sans préjudice du statut des hauteurs du Golan, de Jérusalem Est et des colonies de peuplement israéliennes en Cisjordanie aux termes du droit international. 